\title{
Obesity Etiology
}

\section{Emotional processing in obesity: a systematic review and exploratory meta-analysis}

\author{
J. Fernandes ${ }^{1}$ (D), F. Ferreira-Santos ${ }^{2,3}$ (D), K. Miller ${ }^{4}$ and S. Torres ${ }^{1,3}$ (D)
}

${ }^{1}$ Centre for Psychology at University of Porto (CPUP), Porto, Portugal, ${ }^{2}$ Laboratory of Neuropsychophysiology, University of Porto, Porto, Portugal, ${ }^{3}$ Faculty of Psychology and Educational Sciences, University of Porto, Porto, Portugal, and ${ }^{4}$ School Psychology, School of Education, University of North Carolina at Chapel Hill, Chapel Hill, NC, USA

Received 11 April 2017; revised 10 August 2017; accepted 10 August 2017

Address for correspondence: J Fernandes, Centro de Psicologia da Universidade do Porto, Rua Alfredo Allen, 4200-135, Porto, Portugal. E-mail: jpffernandes@gmail.com

\begin{abstract}
Summary
The role of emotional functioning in the development and maintenance of obesity has been investigated, but the literature is poorly integrated. A systematic review and meta-analysis was performed to explore emotional processing impairments in obesity. PubMed, Web of Knowledge and PsycINFO databases were searched in March 2016, yielding 31 studies comparing emotional processing competencies in individuals with obesity, with or without binge eating disorder (BED), and control groups. Meta-analyses demonstrated that individuals with obesity had higher scores of alexithymia $(d=0.53)$, difficulty in identifying feelings $(d=0.34)$ and externally oriented thinking style $(d=0.31)$, when compared with control groups. On other competencies, patients with obesity, especially those with comorbid BED, reported lower levels of emotional awareness and difficulty in using emotion regulation strategies, namely, reduced cognitive reappraisal and acceptance, and greater suppression of expression. No evidence of impaired ability to recognize emotions in others or verbally express emotions was found. A general emotion-processing deficit in obesity was not supported. Instead, an emotional avoidance style may occur modulating later responses of emotion regulation. Additional research is needed to extend the comprehension of these conclusions and the role of BED in emotional functioning in obesity.
\end{abstract}

Keywords: Emotion processing, meta-analysis, obesity, review.

\begin{abstract}
Abbreviations: BED, Binge eating disorder; BMI, Body mass index; CI, Confidence intervals; CTRL, Control group; DDF, Difficulties in describing feelings; DIF, Difficulties in identifying feelings; EOT, Externally oriented thinking; ER, Emotion regulation; ES, Effect size; NOS, Newcastle-Ottawa Quality Assessment Scale; OB, Group of participants with obesity without assessment of BED; OB-BED, Group of participants with obesity and comorbid binge eating disorder; OB-N/ BED, Group of participants with obesity without comorbid binge eating disorder; TAS, Toronto Alexithymia Scale.
\end{abstract}

Emotional dysfunction seems to play an important role in the development and maintenance of eating disorders (1). In obesity research, several studies have related eating disorders and emotions, arguing that compulsive ingestion of food may constitute an avoidance, suppression or regulation strategy to cope with negative emotions [e.g. $(2,3)]$. However, only during the last decade has research focused on which specific emotional abilities are actually compromised. In the study of this topic, the link with binge eating disorder (BED), an eating disorder defined by recurrent episodes of excessive food intake in a short period, is frequently considered (4). There is evidence that negative emotion serves as a trigger for binge eating episodes in individuals with $\mathrm{BED}$, but not in individuals 
with obesity without comorbid BED (5). Theoretically, it is then possible that emotional difficulties in individuals with obesity may be related to comorbid BED, rather than obesity itself.

Williams et al. (6) provide a useful framework for conceptualizing emotional abilities, which distinguishes between three facets in emotional processing based on a temporal continuum: emotions, thinking and feeling and self-regulation. According to this model, emotions are action tendencies that are triggered automatically, without conscious awareness of the elicited signal. The automatic processing of emotions that occurs in this phase then can be defined as an involuntary and non-conscious perceptual cognitive process that is mobilized by significant emotion cues (7). To assess this process, researchers have used subliminal presentations of emotion stimuli to analyse the tendency to allocate attention to a particular category of stimulus based on its relative affective salience. In obesity, it is not clear whether individuals are more attentive to emotional content (8) or present greater difficulty attending to these features $(9,10)$. Furthermore, it is still unclear whether these impairments occur with all emotional content or are restricted to a particular type of stimuli (e.g. emotional words or facial expressions of emotions) with different valences (positive or negative).

Thinking and feeling, on the other hand, take place at a conscious level and correspond to the subjective experience of emotion. Impairments at this phase may be related to alexithymia, which is considered a deficit in the cognitive-experiential component of the emotion response system (11). Alexithymia is comprised of difficulties in identifying (DIF) and describing feelings (DDF) accompanied by an external oriented thinking style (EOT) (11). The role that alexithymia plays in the onset and maintenance of obesity is not yet well established. Although several studies showed higher levels of alexithymia in individuals with obesity [e.g. $(12,13)]$, others point out that only the subgroup of individuals with obesity and BED displays higher prevalence rates $(14,15)$. In contrast, a more recent study reported no differences between participants with obesity, with or without BED (16). Integration of data considering each alexithymia component separately, in line with its multidimensional nature (11), and the moderator-interaction effect of comorbid BED, depression and anxiety are essential to clarify this association.

The third phase of emotional processing includes the concept of emotion regulation (ER) (6). Although theoretically, the DDF and EOT features of the alexithymia construct are also part of this phase, as by definition alexithymia is a broader concept, we consider it separately. The literature on ER suggests that individuals with obesity have a more limited access to functional/adaptive strategies in comparison with control groups [e.g. (17)]. However, it has not yet been systematized with adaptive and maladaptive ER strategies in individuals with obesity use.

In sum, findings on emotional difficulties in obesity are disperse, and, consequently, it remains unclear to what extent emotional processing impairments exist in obesity. In particular, it is not known (i) whether possible deficits are general to all phases of emotional processing or related to specific competencies; (ii) if emotional difficulties are associated with comorbid BED and (iii) which variables may moderate the relationship between obesity and emotional difficulties. The aim of this study was to conduct a meta-analysis and systematic review of the literature oriented towards these research questions. Four emotional processes were analysed, adapted from Williams et al.'s (6) framework: (i) automatic processing of emotions (attentional bias to emotionally salient stimuli); (ii) alexithymia; (iii) recognition and inference of emotional states in self and others and (iv) the use of ER strategies. The inference of emotional states in self and others, as in (iii), is not covered by the Williams et al.'s model but was added here, as it is considered a relevant, higher level emotional competence that is required when stimuli are ambiguous (18).

To the best of our knowledge, this is the first study to systematize and integrate emotional processing data in obesity. The main focus of previous systematic reviews $(5,19)$ was BED, not obesity, and did not include a wide range of emotional competencies in their analyses. Clarifying the emotional processing impairments in obesity and the conditions under which they are expressed is central to understanding the disease and improving treatment.

\section{Method}

\section{Search strategy}

This study followed guidance from the PRISMA statement (20). In March 2016, PubMed, Web of Knowledge and PsycINFO databases were searched for studies investigating the four previously mentioned emotional processes (search terms are described in Supporting Information, Table S1). If a study was not available in the databases, authors were contacted directly. Papers in English, French, Spanish, Portuguese and Italian were considered for this study.

\section{Eligibility criteria and study selection}

Eligibility criteria were defined according to the PICOS structure (20):

- Population: group of participants with obesity diagnosis. No age restrictions were considered.

- Interventions: No restrictions were defined on the modality of obesity treatment to which the participants 
were to be submitted, except the explicit participation in programs for emotion processing improvement.

- Comparator: control group composed of healthy individuals, community samples or normal-weight participants. Comparisons based on the presence/absence of BED were also eligible.

- Outcomes: Primary outcome variables were the emotional processing competencies categorized according to the adaptation of the Williams et al.'s (6) framework. Secondary outcome variables were depression, anxiety, age, gender and body mass index (BMI).

- Study designs: observational (case-control and crosssectional studies).

The methodological quality of studies, in what concerns to the validity of the assessment instruments, was an additional eligibility criterion. In the assessment of alexithymia, only studies using the Toronto Alexithymia Scale (TAS) - both the previous (TAS-26) and the current version (TAS-20) - were considered, given its wide use and proven validity (21). The TAS-26 included a fourth subscale ('lack of introspection and daydreaming') that was dropped in the latest version, the TAS-20. For this reason, this subscale was eliminated from the analyses. Figure 1 presents the different stages of study selection.

\section{Data extraction}

The following information were extracted from each study into a data extraction sheet: (i) sample size, sociodemographic data (mean age and gender distribution) and mean BMI; (ii) the assessment material (self-report questionnaire, interview or experimental task) and (3) statistical data. The mean, standard deviation and sample size were obtained for self-report and behavioural measures. Possible moderator variables were also coded from papers. Two authors performed data extraction independently, followed by a discussion to resolve discrepancies. A third author was consulted when agreements were not reached. If a study did not report data that had been collected or presented it in a form that could not be used, the original authors were contacted and the missing information was requested. When descriptive statistics were only presented in figures (e.g. in bar graphs), the statistics were estimated by measurement of the figures.

\section{Quality assessment}

The risk of bias of eligible studies was assessed using the Newcastle-Ottawa Quality Assessment Scale (NOS) for case-control studies (22) and the Crombie's items for
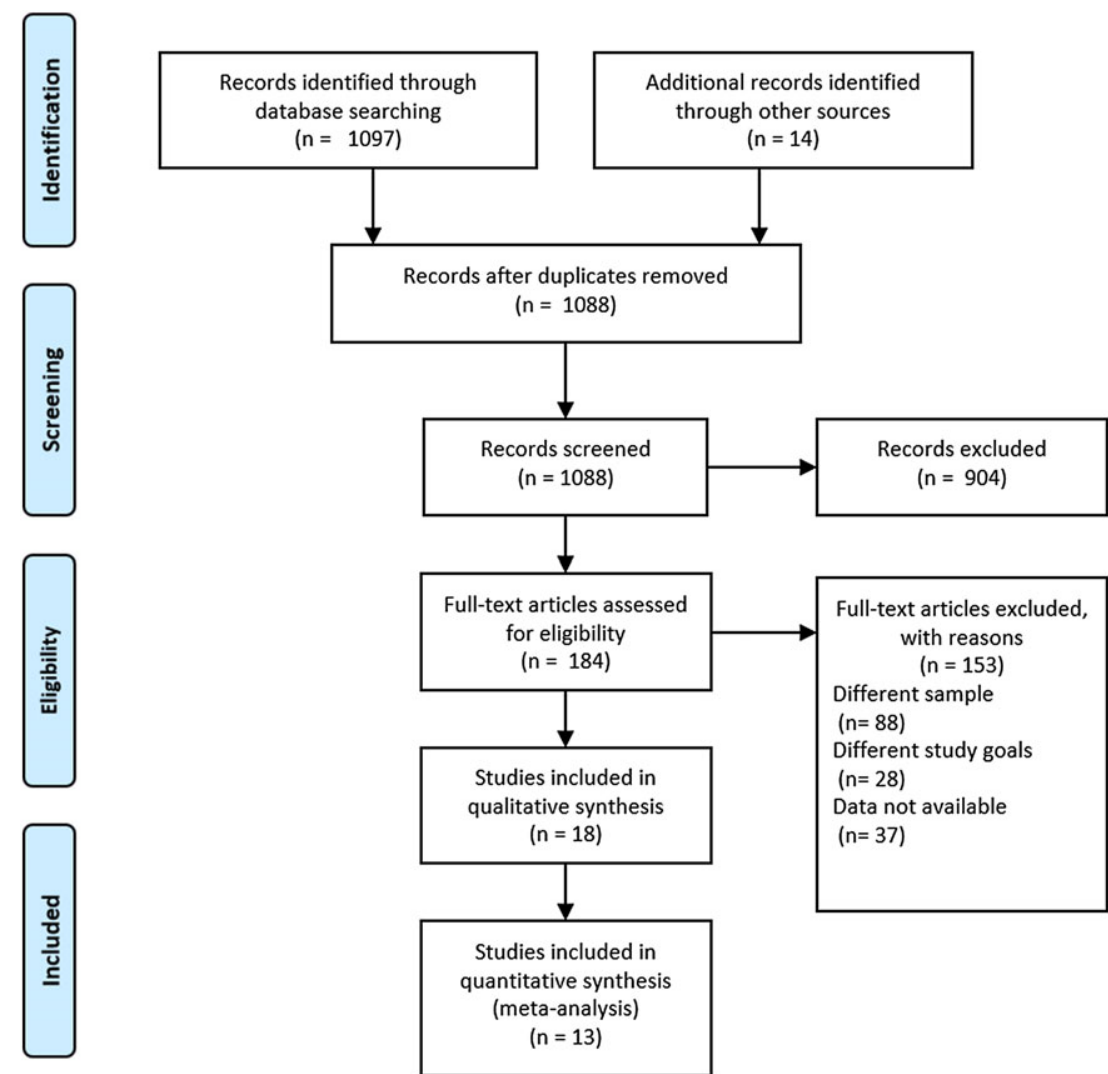

Figure 1 PRISMA flow chart for study inclusion. [Colour figure can be viewed at wileyonlinelibrary.com] 
cross-sectional studies (23). The NOS addresses the quality of case-control studies through three major categories: (i) selection (four items); (ii) comparability (one item) and (iii) exposure (three items). A study can be awarded a maximum of one star for specific items within the selection and exposure categories, and a maximum of two stars can be given for comparability. Nine stars are considered the highest level of quality. NOS scores are categorized into three groups: poor (score 0-3), moderate (score 4-6) and high methodological quality (score 7-9). The Crombie's items contain seven criteria for quality of cross-sectional studies: (i) appropriate design; (ii) adequate description of data; (iii) report response rates; (iv) adequately represent the total sample; (v) clearly stated aims and likelihood of reliable and valid measurements; (vi) statistical significance and (vii) adequate description of analyses. Each item is classified as 'yes' ( 1 point), 'unclear' $(0.5$ point) or 'no' $(0$ point). Quality scores may range from 0 to 7 . All disagreements were resolved by discussion.

\section{Statistical analysis}

Meta-analysis was only performed for alexithymia. Studies on other emotional domains were analysed through systematic review given its reduced number. For each contrast between groups, four separate analyses were performed by combining the effect size (ES) of the alexithymia total score and subscales (DIF, DDF and EOT). Each analysis included between 4 and 10 studies, with four being the minimal requirement for our analyses. The number of studies per meta-analysis in medical research has been typically small, with an overall median of 3 (inter-quartile range 2 to 6) (24). While a larger number is desirable, this size can be acceptable depending on the quality of the studies and total number of cases included in each analysis (25). In our study, methodological quality was taken into consideration in study selection, and there was a minimum of 872 participants with obesity in each analysis.

Mean differences between groups were standardized by calculating Cohen's $d$ using Microsoft Office Excel 2010. Cohen's $d$ ES is the difference between the two raw means of the groups divided by the pooled standard deviation (26). ES and 95\% confidence intervals (CIs) were reported. A positive Cohen's $d$ ES would indicate higher levels of alexithymia and factors in the group of participants with obesity. When considering contrasts based on the presence/absence of BED, a positive ES would indicate higher emotional difficulties in the group with BED. Analyses were conducted using the 'metafor' add-on package for meta-analyses (version 1.9-5) (27) with $R$ (version 3.1.2; $\mathrm{R}$ Core Team, 2014). Due to the limited number of studies and potential issues with heterogeneity, random effect models were used for the meta-analytic combination of ES (28). The homogeneity of ES was also formally assessed using the $Q$ statistic and the $I^{2}$ index to measure the magnitude of the heterogeneity (29). Publication bias was assessed through Egger's test (30). The effects of the following moderator variables were analysed: assessment measure (TAS-26 or TAS-20), depression, anxiety (symptomatology or trait), mean age, proportion of female participants in the sample and BMI.

\section{Results}

\section{Description of included studies}

The reviewed studies showed that the alexithymia construct has been a topic of interest in obesity research since the mid 1990s, while other emotional competencies have only gained attention from early years of the 21st century. In what concerns study design, out of 31studies, 21were considered case-control and 10 cross-sectional. Three types of contrasts were found in case-control studies: (i) group of participants with obesity without assessment of BED (OB) vs. control group (CTRL) ( $n=14)$; (ii) group of participants with obesity and comorbid BED (OB-BED) vs. group of participants with obesity and without comorbid BED (OB-N/ BED) $(n=9)$ and (iii) group of participants with obesity and comorbid BED (OB-BED) vs. control group (CTRL) $(n=2)$.

CTRL groups were mainly composed of normal-weight participants (BMI varied between 21 and $23 \mathrm{~kg} \mathrm{~m}^{-2}$ ); only one study used a community-based sample with no restrictions for BMI (31). Participant's BMI in OB groups ranged between 31 and $47 \mathrm{~kg} \mathrm{~m}^{-2}$.

Only adult samples were included, ranging from 28 to 49 years of age. Twelve studies included participants of both genders, despite of an overrepresentation of female gender. It should be mentioned that three studies were found using children samples between 10 and 13 years old $(8,32,33)$, distributed among different emotional competencies; however, as they cannot be representative of children population due to their small number, they were excluded.

Self-report was the most commonly used method for assessing emotional competencies and possible moderators. Only studies on automatic processing and emotion recognition used direct, experimental tasks based on visual material. The diagnosis of BED was determined by selfreport $(n=4)$ or semi-structured interview $(n=8)$.

Moderator effects of BMI, gender and age were only reported in alexithymia studies. The moderator effect of depression was reported in nine studies, specifically alexithymia $(n=6)$, automatic processing $(n=2)$ and ER $(n=1)$. The effect of anxiety was only analysed for alexithymia $(n=1)$, automatic processing $(n=1)$ and ER $(n=1)$. 


\section{Quality assessment}

The number of stars on the NOS for each case-control study ranged from 4 (moderate quality) to 7 (high quality), with the median score of 6 . All studies were truly representative of cases. Controls were selected from community samples $(n=16)$ or within the same population $(n=6)$ when OB-BED vs. OB-N/BED comparison was considered. Two studies did not describe the context in which the participants were recruited. Only five studies mentioned that controls had no history of disease. With regards to comparability, all studies controlled at least for one type of variable (e.g. age, gender or education) and presented a secure record to ascertain comparability between groups (e.g. BMI calculation) or structured interview/written selfreport to assess BED comorbidity. All but one study did not report the non-response rate. The details of the quality evaluation for each enrolled study are presented in Supporting Information, Table S2.

The scores of cross-sectional studies assessed by Crombie's items ranged from 5 to 6 indicating good quality (Supporting Information, Table S3). 'Unclear' or 'no' classification was mainly assigned to the items relative to the report of response rates and the adequate representativeness of the total sample.

\section{Meta-analysis of alexithymia}

Table 1 reports meta-analytic data. The main characteristics of studies included in the meta-analysis and systematic review (Table S4) and the forest plots performed according to each group contrast (Fig. S1) are presented in Supporting Information.

\section{Alexithymia total score}

Ten studies were included in the meta-analysis comparing OB and CTRL groups in total alexithymia scores. Higher levels of alexithymia were found in $\mathrm{OB}, d=0.53,95 \% \mathrm{CI}$
$[0.33,0.72], p<0.001$. Significant heterogeneity was found across studies with no evidence of publication bias ( $p=0.418$ ). Comparing OB-BED vs. OB-N/BED samples, five studies were considered and showed that OB-BED had higher alexithymia scores, $d=0.47,95 \%$ CI $[0.17,0.78]$, $p=0.002$. The small number of studies considered in this set of analyses did not allow for assessing publication bias, and no significant heterogeneity was found across studies. Of note, this result was common to all alexithymia subscales, in what concerns to OB-BED vs. OB-N/BED.

\section{Difficulties in identifying feelings}

Twelve studies were included in this analysis. Eight of these were considered in the OB vs. CTRL set and found greater DIF scores in OB, $d=0.34,95 \%$ CI $[0.16,0.51]$, $p<0.001$. There was significant heterogeneity across studies and no evidence of publication bias $(p=0.109)$. Considering the second set of analyses, DIF was higher in people with OB-BED relative to OB-N/BED $, d=0.63,95 \% \mathrm{CI}$ $[0.32,0.95], p<0.001$.

\section{Difficulties in describing feelings}

Twelve studies examined the DDF dimension. Eight studies were included in the OB vs. CTRL set and revealed that, in spite of increased scores observed in $\mathrm{OB}$ samples, the differences were not significant, $d=0.20,95 \%$ CI $[-0.01,0.41]$, $p=0.06$. Significant heterogeneity was found across studies and no evidence of publication bias $(p=0.55)$. For the second set of analyses, four studies were included and showed that OB-BED presented negligible differences in comparison with OB-N/BED in DDF, $d=0.10,95 \%$ CI $[-0.27,0.46]$, $p=0.60$.

\section{Externally oriented thinking}

Eleven studies were included with regards to the EOT dimension. In the OB vs. CTRL analysis, which included seven studies, the direction of effects showed increased levels of concrete thinking in $\mathrm{OB}, d=0.31,95 \%$ CI [0.05,

Table 1 Statistical outcomes for all meta-analyses

\begin{tabular}{|c|c|c|c|c|c|c|c|}
\hline & $k$ & $d$ & $p_{d}$ & $95 \% \mathrm{Cl}$ & $Q$ & $p_{Q}$ & $1^{2}$ \\
\hline \multicolumn{8}{|c|}{ OB vs. CTRL } \\
\hline TAS & 10 & 0.53 & $<0.0001$ & {$[0.33,0.72]$} & 49.531 & $<0.0001$ & 72.49 \\
\hline DIF & 8 & 0.34 & $<0.001$ & {$[0.16,0.51]$} & 23.926 & $<0.001$ & 70.69 \\
\hline DDF & 8 & 0.20 & 0.006 & {$[-0.01,0.41]$} & 39.652 & $<0.0001$ & 79.54 \\
\hline EOT & 7 & 0.31 & 0.002 & {$[0.05,0.57]$} & 40.330 & $<0.0001$ & 84.76 \\
\hline \multicolumn{8}{|c|}{ OB-BED vs. OB-N/BED } \\
\hline TAS & 5 & 0.47 & $<0.001$ & {$[0.17,0.78]$} & 9.842 & 0.043 & 60.37 \\
\hline DIF & 4 & 0.63 & $<0.0001$ & {$[0.32,0.95]$} & 7.370 & 0.061 & 60.43 \\
\hline DDF & 4 & 0.10 & 0.598 & {$[-0.27,0.46]$} & 11.848 & $<0.01$ & 71.53 \\
\hline EOT & 4 & -0.001 & 0.924 & {$[-0.24,0.21]$} & 4.348 & 0.226 & 26.83 \\
\hline
\end{tabular}

$\mathrm{OB}$, obesity without assessment of binge eating disorder; CTRL, control group without assessment of binge eating disorder; OB-BED, obesity with binge eating disorder; OB-N/BED, obesity without comorbid binge eating disorder; TAS, Toronto Alexithymia Scale; DIF, difficulty in identifying feelings; DDF, difficulty in describing feelings; EOT, externally oriented thinking; $\mathrm{Cl}$, confidence interval; $k$, number of samples; $p_{d}$, significance of $d$; $Q$, Cochran's measure of homogeneity; $p_{Q}$, significance of $Q ; F^{2}$, percentage of effect size variability explained by heterogeneity between samples. 
0.57], $p=0.02$. There was significant heterogeneity across studies and no evidence of publication bias $(p=0.363)$. No differences between OB-BED and OB-N/BED were found with regards to EOT, $d=-0.01,95 \%$ CI $[-0.24$, $0.21], p=0.92$.

\section{Moderator variables}

No effects were found for the assessment measure used in the studies (TAS-26 or TAS-20), depression, mean age, gender distribution or BMI that could explain variation between studies. Only one study controlled for anxiety, which limited the analysis of this variable.

\section{Systematic review of alexithymia}

Studies in this section enable a better understanding of the relationship of alexithymia with $\mathrm{BED}$, anxiety, gender and BMI, and they were not included in the meta-analysis because different group contrasts were used. Groups of $\mathrm{OB}$ with and without alexithymia were compared in two studies, concluding when $\mathrm{OB}$ with greater levels of alexithymia presented higher scores of BED (42) but not of anxiety (16). Gender differences in alexithymia prevalence were assessed in two studies $(31,43)$, which did not find significant differences between men and women with obesity. However, it should be noted that higher scores were endorsed by men in the EOT dimension $(31,43)$, while women reported higher scores in DIF (31). The effect of BMI reduction on the variation of alexithymia was tested in two studies that compared two samples of participants with obesity: one composed of patients seeking weight loss surgery and other with patients that were already submitted to treatment (biliopancreatic diversion (44) or conservative weight loss intervention (45)). No significant differences between groups were found. Similarly, in a correlational study, Troisi et al. (46) reported no significant association between BMI and TAS total score.

\section{Systematic review of automatic processing of emotions}

Two studies examined automatic processing of emotions, each using different experimental tasks to assess this emotional process. They used an affective priming task with positive and negative emotional stimuli of schematic faces (9) and adjective words (10), observing greater difficulties on being attentive to emotional stimuli in $\mathrm{OB}$ compared with CTRL. This data is suggestive of problems in being attentive to emotions. Comparisons of the valence were only reported in one study (9) with higher difficulties towards negative facial expressions observed. Difficulties with the automatic processing of emotions appear to occur independently of self-reported depression $(9,10)$ and anxiety (9).

\section{Systematic review of recognition and inference of emotions in self and others}

Studies herein were those that analysed the ability to recognize emotions in others, implemented with the use of visual material, and those that assessed the ability to infer mental states in self and others. With regards to emotion recognition in others, one study used a sample of female adults with obesity and found no differences between OB and CTRL in the correct recognition of facial expressions presented on a computer screen (47). When inferring emotional states in self and others, greater difficulties were found in OB in comparison with CTRL (48). In analysing the results from the two subscales provided by the Levels of Emotional Awareness Scale (Self and Others), the OB showed more difficulties in the ability to infer the emotional states of others compared with their own emotional states.

\section{Systematic review of emotion regulation}

Eight studies were found with regards to ER, addressing the general difficulty in accessing functional strategies or the specific use of certain ER strategies, classified as adaptive (cognitive reappraisal, acceptance, distraction) or maladaptive (suppression of feelings, rumination). Data relating to general access to functional ER strategies indicated less ability both in OB (17) and OB-BED (49) relative to CTRL. With respect to specific adaptive strategies, studies reported a decreased use of cognitive reappraisal in OB-BED when compared with OB-N/BED (50,51) and CTRL (49,52) groups. Also, the use of strategies to accept feelings and situations proved to be reduced in OB-BED in comparison with CTRL (49). However, it should be noted that comparative analysis including all participants with obesity in the same group, independent of having BED or not, failed to detect differences in cognitive reappraisal (40), distraction (53) and acceptance of emotions (17), relative to CTRL group.

Six studies assessed maladaptive strategies. Expressive suppression was higher in OB-BED compared with OB-N/ $\operatorname{BED}(50,51)$ and CTRL groups $(49,52)$. One study comparing OB with CTRL also found higher scores in OB (40). The use of this strategy was not related to trait anxiety in OBBED (52). In contrast, after controlling for depression, one study showed no differences between OB-BED, OB-N/BED and CTRL in anger suppression (54). Comparisons of CTRL with OB-BED, for thought suppression (49), and $\mathrm{OB}$, for rumination (53), did not reveal significant differences.

\section{Discussion}

This meta-analytic and systematic review sought to examine and summarize what is known to date about emotion processing difficulties in people with obesity. The results of 
the meta-analysis indicated that alexithymia seems to reach higher levels in people with $\mathrm{OB}$, when compared with CTRL groups. Analysis of the three dimensions of the alexithymia construct suggests how they might behave differently in obesity. Increased scores in DIF and EOT factors, but not in DDF, were found in individuals with this disease. These findings are particularly surprising in what concerns DDF. As DIF tended to be higher in OB, the same was expected to happen with DDF, given that verbal report of feelings is contingent on the ability to successfully identify them (55). Two arguments can be made on the basis of this result. Firstly, emotional reporting can occur even if individuals are completely unaware of their emotional state. Individuals may be able to report and label inner discomfort in terms of the cognitions available to them, even not distinguishing the internal cues that allow the experiential dimension of each emotion, as previously documented in eating disorders (56). Secondly, if there is a true DDF, the difficulties may not be self-evident. We speculate that some traits, such as extraversion, which has been linked to obesity (57), can increase the individuals' perception of being able to express emotions, considering that they view themselves as sociable. However, sociability and talkativeness do not necessarily imply communicating feelings. The discussion of this topic highlights the limitation of using self-reported data in the assessment of emotional competencies, considering the potential importance of self-awareness needed for self-report. Due to lack of introspective access to implicitly assessed representations, results from self-report measures should be interpreted with caution (58).

In the literature, alexithymia has been associated with a wide range of emotional impairments $(59,60)$, and our study reinforces this perspective. Along the three phases of emotional processing, several difficulties were found, during systematic review. Research using affective priming tasks showed that $\mathrm{OB}$ participants had more difficulties being attentive to emotions than CTRL. Less pronounced affective reactions to primed stimuli in $\mathrm{OB}$ may indicate that these individuals tend to subconsciously avoid emotions. However, it has been hypothesized that affect-biased attention is a result of personal experiences, which might regulate or even modulate subsequent conscious emotional responses and experiences (7). These findings stress the relevance of intervention in affect-biased attention in order to regulate emotional responses to later stressful events. A preliminary finding, due to the small number of studies, was the difficulty with inference of emotional states. Particularly reduced is the capacity of OB groups to represent others' emotional experience, which is in line with the impairments in empathy typically found in alexithymic individuals $(11,59)$. Also, the access to functional strategies to deal with emotions proved to be limited in patients with obesity, especially those with comorbid BED. They seem to rely more on the suppression of ongoing emotional behaviour, with less reported use of adaptive strategies, such as cognitive reappraisal and acceptance. According to the literature, this pattern of decreased use of adaptive cognitive change and increased use of maladaptive emotion suppression strategies is associated with less healthy patterns of affect, social functioning and well-being (60).

However, and despite these difficulties, it should be noted that some other competencies involved in emotional processing were intact for individuals with obesity. There is no evidence for problems with recognition of emotions through facial and verbal expression of emotions. Moreover, in what concerns to the use of maladaptive ER strategies, only expressive suppression proved to be more common in obesity. No increased use of distraction or rumination was found. These two last ER strategies are included in one of the first emotion regulatory processes, referred as attentional deployment. In turn, emotion suppression is a strategy included in the response modulation phase, which occurs later, after response tendencies have been initiated (61). In light of this, it seems that maladaptive strategies tend to be used in the response-focused ER phase, rather than in the antecedent-focused ER phase.

In sum, although preliminary in its conclusions, this study highlights the presence of emotion functioning difficulties in obesity, despite limited extension to all emotional competencies. The fact that some skills seem to be preserved suggests that there may not be a general emotion-processing deficit in obesity. We are more inclined to hypothesize that emotion difficulties in these patients are rooted in an emotion avoidance process, in line with the association between suppression of stressful experiences and weight stigmatization documented in the literature (62). In the frame of a temporal continuum of emotion (6), the increased tendency to avoid emotions found in individuals with obesity can impact their ability to acknowledge emotional states and, consequently, to adaptively cope with them. Difficulties in ER might include a lack of adaptive strategies, the presence of maladaptive strategies or both (52).

Binge eating may also constitute a maladaptive strategy to regulate emotions, providing short-term distraction from affect (2). There is some evidence in the literature that people with BED symptoms are less able to identify and deal with emotions (19), and our findings are congruent with this view. Studies included in the present review revealed that lower emotional awareness and difficulties in ER strategies (namely, greater expressive suppression and reduced cognitive reappraisal and acceptance) were generally associated with the presence of BED in patients with obesity. Negative emotion, which can easily arise in a context of body dissatisfaction, has been related to binge eating in individuals with BED (5), and this relationship seems to be partially explained by deficits in differentiating between feelings and sensations of hunger/satiety (63). Binge eating behaviour can emerge to compensate for ER 
difficulties, but it can itself potentiate them by limiting the use of more efficient ER strategies (5). The direction of causality between emotional difficulties and BED in obesity is not yet defined, and it is a topic for clarification in future longitudinal studies. So far, we must only conclude that most difficulties in identifying and regulating emotions do not appear to be associated with obesity per se, but rather with eating disorder psychopathology, as previously hypothesized (19). This conclusion is not valid for EOT, which proved to be increased in patients with obesity, both with or without BED. This focus on external events can be a stable feature in this disease explained by an overresponsiveness to external stimuli, both food and non-food related, as postulated by the Schachter's (64) externality theory of obesity. Overall, these data suggest that not all alterations in emotional processing in obesity are attributable to comorbid BED. In order to identify which emotional difficulties are better explained by BED, the effect of this diagnosis should be controlled for in future studies on automatic processing of emotions, emotion recognition and inference and some ER strategies (e.g. distraction, rumination, problem-solving and avoidance). Depending on their conclusions, it may be appropriate to treat individuals with obesity, with and without BED, as distinct groups.

The study of the combined effect of BED and depression is another topic of interest. The relevance of this interaction is stressed by the literature on ER by suppression of emotional expression. This strategy was more frequent in OBBED relative to OB-N/BED $(49,50)$, but when depression was controlled for, this difference was not detected (54). From the theoretical point of view, depression can offer one explanation for increased emotion suppression in those who binge eat. Thus, assuming that psychiatric comorbidity in obesity is high (14), especially when associated with BED (16), we stress the need to explore the combined effect of these variables in further studies.

Even data exploring the single effect of depression or anxiety on emotional difficulties were scarce and were not suggestive of a significant association. The role of depression was only explored in studies on alexithymia and automatic processing, with no significant moderator effects detected. Concerning anxiety, findings also revealed no significant impact on the variation of alexithymia, automatic processing of emotions and expressive suppression.

Other moderator variables, such as age, BMI and gender, were only explored in literature in relation to alexithymia. BMI and alexithymia were not significantly associated, in spite of increased scores of alexithymia found in OB relative to CTRL. There may be a stable degree of alexithymia in the normal-weight range and within $\mathrm{OB}$ samples. With regards to gender, differences between men and women were reported in the DIF and EOT dimensions. In particular, gender differences in EOT have also been reported in studies with non-clinical samples [e.g. 63], suggesting that there might be a gender effect on this alexithymia subscale, independent of the obesity condition. This hypothesis requires further confirmation.

\section{Limitations}

Findings should be understood in the context of limitations related to both the literature and this review. One limitation of the literature is the unbalanced number of studies for each emotional competence analysed. The majority of studies focused on alexithymia, with a significant lack of replication studies in other emotional skills limiting the conclusions that can be drawn. Understanding the effect of BED in impaired emotional functioning is also very preliminary, in particular in deficits beyond alexithymia. Many studies included in this review failed to differentiate OB-N/BED from OB-BED with regards to specific emotional competencies. In addition, most studies did not appropriately control for other possible moderator factors, such as sociodemographic variables, comorbid anxiety and depression, psychiatric medication use and cognitive functioning.

Methodological issues have also impacted this review. The diversity of experimental paradigms (e.g. affective priming or Stroop tasks) and different stimuli (e.g. video or photographs) made the comparison between studies difficult. There is also some heterogeneity in OB samples, which hampers the comparison of data. $\mathrm{OB}$ samples are normally composed of treatment-seeking patients, but the modality of treatment is not controlled for. Thus, there is a possibility that interventions delivered prior to assessment may have focused on emotional competencies.

As with all reviews, the decisions made over inclusion criteria are open to criticism. Also, the proposed theoretical framework used is this study had some limitations. Williams et al.'s (6) model is not wholly sufficient in defining emotional processing, as it is mainly oriented to personal emotions and not others' emotions. As the aim of this review was not testing this model, but rather using it as a guide for organizing current research, it was decided to include additional emotional competencies to this review, ad hoc, not covered by Williams et al. (6). In particular, the recognition and inference of emotional states in others consider that these competences are crucial to establishing adaptive social interactions.

\section{Conclusion}

This study does not support the hypothesis of a general emotion-processing deficit in obesity, covering all emotional skills. No evidence of impaired ability to recognize emotions in others or verbally express emotions was found. Overall, the emotion difficulties observed in these patients seem to be rooted in an emotion avoidance process, which starts at 
a sub-conscious level, modulating subsequent conscious emotional experiences and responses. Along the continuum of emotional processing, we found evidence of lower emotional awareness and difficulties in ER. More limited access to adaptive strategies to deal with emotions and the increased use of expressive suppression were the main findings in the emotion regulatory process. It remains difficult, however, to determine whether alterations in emotion processing in obesity are attributable to BED, as its effect was not controlled for in the study of several emotional difficulties. Far more research is required to determine the nature of impaired emotional functioning in obesity, and here, we offered some guidelines to accomplish it.

\section{Conflict of interest statement}

No conflict of interest was declared.

\section{Acknowledgements}

This research was funded by Fundação para a Ciência e Tecnologia (FCT - Foundation for Science and Technology) and Centre for Psychology at University of Porto (CPUP; UID/PSI/00050/2013).

\section{Supporting information}

Additional Supporting Information may be found online in the supporting information tab for this article. https://doi. org/10.1111/obr.12607

Table S1. Outline of constructs and search terms used in the current study

Table S2. Appraisal of methodological quality of the casecontrol studies using the Newcastle-Ottawa Scale

Table S3. Appraisal of methodological quality of the crosssectional studies using Crombie's items

Table S4. Characteristics of the studies included in the meta-analysis and systematic review

\section{References}

1. Schmidt U, Treasure J. Anorexia nervosa: valued and visible. A cognitive-interpersonal maintenance model and its implications for research and practice. Br J Clin Psychol. 2006; 45: 343-366.

2. Gianini L, White M, Masheb R. Eating pathology, emotion regulation, and emotional overeating in obese adults with binge eating disorder. Eat Behav. 2013; 14: 309-313.

3. Macht M. How emotions affect eating: a five-way model. Appetite. 2008; 50: 1-11.

4. American Psychiatric Association. Diagnostic and statistical manual of mental disorders, 5th edn. American Psychiatric Press: Washington, 2013.

5. Leehr E, Krohmer K, Schag K, Dresler T, Zipfel S, Giel K. Emotion regulation model in binge eating disorder and obesity - a systematic review. Neurosci Biobehav R. 2015; 49: 125-134.
6. Williams L, Gatt J, Hatch A et al. The integrated model of emotion, thinking and self-regulation: an application to the 'paradox of aging'. J Integr Neurosci. 2008; 7: 367-404.

7. Todd R, Cunningham W, Andersen A, Thompson E. Affect-biased attention as emotion regulation. Trends Cogn Sci. 2012; 16(7): 365-372.

8. Braet C, Crombez G. Cognitive interference due to food cues in childhood obesity. J Clin Child Psychol. 2003; 32: 32-39.

9. Cserjési R, Vermeulen N, Lénárd L, Luminet O. Reduced capacity in automatic processing of facial expression in restrictive anorexia nervosa and obesity. PsychiatryRes. 2011; 188: 253-257. 10. Wegener I, Wawrzyniak A, Imbierowicz K, Conrad R. Evidence for attenuated affective processing in obesity. Psychol Rep. 2008; 103: 35-47.

11. Taylor G, Bagby R, Parker J. Disorders of affect regulation: alexithymia in medical and psychiatric illness. Cambridge University Press: New York, 1997.

12. Da Ros A, Vinai P, Gentile N, Forza G, Cardetti S. Evaluation of alexithymia and depression in severely obese patients not affected by eating disorders. Eat Weight Disord. 2011; 16: 24-29.

13. Noli G, Cornicelli M, Mariani G, Carlini F, Scopinaro N, Adami G. Alexithymia and eating behaviour in severely obese patients. J Hum Nutr Diet. 2010; 23: 616-619.

14. Pinna F, Lai L, Pirarba $S$ et al. Obesity, alexithymia and psychopathology: a case-control study. Eat Weight Disord. 2011; 16: 164-170.

15. Pinaquy S, Chabrol H, Simon C, Louvet J, Barbe P. Emotional eating, alexithymia, and binge eating disorder in obese women. $\mathrm{Ob}$ Res. 2003; 11(2): 195-201.

16. Zak-Golab A, Tomalski $\mathrm{R}$, Bak-Sosnowska $\mathrm{M}$ et al. Alexithymia, depression, anxiety and binge eating in obese women. Eur J Psychiat. 2014; 27(3): 149-159.

17. Fereidouni F, Atef-Vahid M, Lavasani F, Orak R, Klonsky E, Pazooki A. Are Iranian obese women candidate for bariatric surgery different cognitively, emotionally and behaviorally from their normal weight counterparts? Eat Weight Disord. 2015; 20: 397-403.

18. Oldershaw A, Hambrook D, Stahl D, Tchanturia K, Treasure J, Schmidt U. The socio-emotional processing stream in anorexia nervosa. Neurosci Biobehav R. 2011; 35: 970-988.

19. Kittel R, Brauhardt A, Hillbert A. Cognitive and emotional functioning in binge eating disorder: a systematic review. Int J Eat Disorder. 2015; 48(6): 535-554.

20. Moher D, Liberati A, Tetzlaff J, Altman DG, Group TP. Preferred reporting items for systematic reviews and metaanalyses: the PRISMA statement. PLoS Med 2009; 6: 1-6.

21. Lumley M, Lynn C, Neel BA. The assessment of alexithymia in medical settings: implications for understanding and treatment health problems. J Pers Asses. 2007; 89(3): 230-246.

22. WellsG, SheaB, O'Connel, Robertson J, PetersonJ, WelschV, et al.The Newcastle-Ottawa Scale (NOS) for assessing the quality of nonrandomized studies in meta-analysis. [accessed on 26 July 2017]. Available athttp://www.ohri.ca/programs/clinical_epidemiology/oxford.asp.

23. Crombie I. The pocket guide to critical appraisal: a handbook for health care professionals. BMJ: London, 1996.

24. Davey J, Turner R, Clarke M, Higgins J. Characteristics of meta-analysis and their component studies in the Cochrane Database of Systematic Reviews: a cross-sectional, descriptive analysis. BMC Med Res Methodol. 2011; 11: 160-171.

25. Vrijheid M, Martinez D, Manzanares S et al. Ambient air pollution and risk of congenital anomalies: a systematic review and meta-analysis. Environ Health Persp. 2011; 119(5): 598-506. 
26. Cohen J. A power primer. Psychol Bul. 1992; 112: 155-159. 27. Viechtbauer W. Conducting meta-analysis in $\mathrm{R}$ with metaphor package. J Stat Softw. 2010; 36(3): 1-48.

28. Hedges L, Vevea J. Fixed and random effects models in metaanalysis. Psychol Methods. 1998; 3(4): 486-404.

29. Higgins J, Thompson S, Deeks J, Altman D. Measuring inconsistency in meta-analysis. BMJ. 2003; 327: 557-560.

30. Egger M, Smith G, Schneider M, Minder C. Bias in meta-analysis detected by a simple, graphical test. BMJ. 1997; 315: 629-634.

31. Elfhag K, Lundh L. TAS-20 alexithymia in obesity, and its links to personality. Scand J Psychol. 2007; 48: 391-398.

32. Baldaro B, Rossi N, Caterina R, Codispoti M, Balsamo A, Trombini G. Deficit in the discrimination of nonverbal emotions in children with obesity and their mothers. Int J Obes. 2003; 27: 191-195. 33. Surcinelli P, Baldaro B, Balsamo A, Bolzani R, Gennari M, Rossi N. Emotion recognition and expression in young obese participants: preliminary study. Percept Mot Skills. 2007; 105: 477-482. 34. De Lenclave M, Florequin C, Bailly D. Obésité, alexithymie, troubles psychopathologiques et binge eating: étude comparative entre 40 obèses et 32 témoins. L’Encéphale. 2001; XXVII: 343-350 French.

35. Fukunishi I, Kaji N. Externally oriented thinking of obese men and women. Psychol Rep. 1997; 80: 219-224.

36. Molinari E, Morosin A, Riva G. Alessitimia e psicopatologia in un campione clinico di soggetti obesi. Minerva Psichiatrica. 1995; 36: 133-138 Italian.

37. Morosin A, Riva G. Alexithymia in a clinical sample of obese women. Psychol Rep. 1997; 80: 387-394.

38. Zanasi M, Giannoudas I, Testoni F, Melis M, Chiaramonte C, Siracusano A. Dreaming activity in bariatric surgery candidates. Dreaming. 2014; 24(3): 217-231.

39. Zeeck A, Stelzer N, Linster H, Joos A, Hartmann A. Emotion and eating in binge eating disorder and obesity. Eur Eat Disord Rev. 2011; 19: 426-437.

40. Zijlstra H, van Middendorp H, Devaere L, Larsen JK, van Ramshorst B, Geenen R. Emotion processing and regulation in women with morbid obesity who apply for bariatric surgery. Obes Surg. 2012; 20: 1-13.

41. De Zwaan M. Binge eating disorder and obesity. Int J Obes Relat Metab Disord. 2001; 25(Suppl 1): S51-S55.

42. Carano A, Berardis D, Campanella D et al. Alexithymia and suicide ideation in a sample of patients with binge eating disorder. J Psychiatr Pract. 2012; 18(1): 5-11.

43. Larsen J, van Strien T, Eisinga R, Engels R. Gender differences in the association between alexithymia and emotional eating in obese individuals. J Psychosom Res. 2006; 60: 237-243.

44. Adami G, Campostano A, Ravera G, Leggieri M, Scopinaro N. Alexithymia and body weight in obese patients. Behav Med. 2001; 27: 121-126.

45. Gradaschi R, Noli G, Cornicelli M, Camerini G, Scopinaro N, Adami G. Do clinical and behavioral correlates of obese patients seeking bariatric surgery differ from those of individuals involved in conservative weight loss programme? J Hum Nutr Diet. 2013; 26(1): 34-38.

46. Troisi A, Scucchi S, San Martino L, Montera P, d'Amore A, Moles A. Age specificity of the relationship between serum cholesterol and mood in obese women. Physiol Behav. 2001; 72: 409-413.
47. Bergmann S, Klitzing K, Keitel-Korndorfer A et al. Emotional availability, understanding emotions, and recognition of facial emotions in obese mothers with young children. J Psychossom Res. 2016; 80: 44-52.

48. Rommel D, Nandrino J, Ducro C, Andrieux S, Delecourt F, Antoine P. Impact of emotional awareness and parental bonding on emotional eating in obese women. Appetite. 2012; 59: 21-26. 49. Svaldi J, Griepenstroh J, Tuschen-Caffier B, Ehring T. Emotion regulation deficits in eating disorders. A marker of eating pathology or general psychopathology? Psychiatr Res. 2012; 197: 103-111. 50. Svaldi J, Caffier D, Tuschen-Caffier B. Emotion suppression but not reappraisal increases desire to binge in women with binge eating disorder. Psychother Psychossom. 2010; 79: 188-190.

51. Svaldi J, Tuschen-Caffier B, Trentowska M, Caffier D, Naumann E. Differential caloric intake in overweight females with and without binge eating: effects of a laboratory-based emotion regulation training. Behav Res Ther. 2014; 56: 39-46.

52. Danner U, Sternheim L, Evers C. The importance of distinguishing between different eating disorders (sub)types when assessing emotion regulation strategies. Psychiatr Res. 2014; 215: 727-732.

53. Appelhans B, Whited M, Schneider K, Oleski J, Pagoto S. Response style and vulnerability to anger-induced eating in obese adults. Eat Behav. 2011; 12(1): 9-14.

54. Fassino S, Leombruni P, Pierò A, Abbate-Daga G, Rovera G. Mood, eating attitudes, and anger in obese women with and without binge eating disorder. J Psychossom Res. 2003; 54: 559-566.

55. Bagby R, Taylor G, Parker J. The twenty-item Toronto Alexithymia Scale: II. Convergent, discriminant, and concurrent validity. J Psychosom Res. 1994; 38: 33-40.

56. Torres S, Guerra MP, Lencastre L et al. Cognitive processing of emotions in anorexia nervosa. Eur Eat Disord Rev. 2011; 19(2): 100-111.

57. Sutin A, Ferrucci L, Zonderman A, Terracciano A. Personality and obesity across the adult lifespan. J Pers Soc Psychol. 2011; 101(3): 579-592.

58. Hofmann W, Gawronski B, Gschwendner T, Le H, Schmitt M. A meta-analysis on the correlation between the implicit association test and explicit self-report measures. Pers Soc Psychol B. 2005; 31(10): 1369-1385.

59. Grynberg D, Chang B, Corneille $\mathrm{O}$ et al. Alexithymia and the processing of emotional facial expressions (EFEs): systematic review, unanswered questions and further perspectives. PLoS One. 2012; 7(8) e42429.

60. Pandey R, Saxena P, Dubey A. Emotion regulation difficulties in alexithymia and mental health. Eur J Psychol. 2011; 7(4): 604-623.

61. Gross J. The emerging field of emotion regulation: an integrative review. Rev Gen Psychol. 1998; 2(3): 271-299.

62. Lillis J, Hayes S, Bunting K, Masuda A. Teaching acceptance and mindfulness to improve the lives of the obese: a preliminary test of a theoretical model. Ann Behav Med. 2009; 37(1): 58-69.

63. Van Strien E, Engels R, Van Leeuwe J, Snoek H. The Stice model of overeating: tests in clinical and non-clinical samples. Appetite. 2005; 45: 205-213.

64. Schachter S. Some extraordinary facts about obese humans and rats. Am Psychol. 1971; 26: 129-144. 